\title{
NMR and Pattern Recognition Can Distinguish Neuroinflammation and Peripheral Inflammation
}

\author{
Agnieszka Smolinska, ${ }^{\dagger}$ Amos Attali, ${ }^{\neq}$Lionel Blanchet, ${ }^{\dagger}$ Kirsten Ampt, ${ }^{\dagger}$ Tinka Tuinstra, ${ }^{\ddagger}$ Hans van Aken, ${ }^{\neq}$ \\ Ernst Suidgeest, ${ }^{\ddagger, \#}$ Alain J. van Gool, ${ }^{\S}$ Theo Luider, ${ }^{\prime \prime}$ Sybren S. Wijmenga, ${ }^{\dagger}$ and Lutgarde M.C. Buydens ${ }^{*,+}$ \\ ${ }^{\dagger}$ Institute for Molecules and Materials, Radboud University Nijmegen, Nijmegen, The Netherlands \\ ${ }^{\ddagger}$ Abbott Healthcare Products B.V., Weesp, The Netherlands \\ ${ }^{\S}$ Molecular Profiling, MSD, Singapore, Singapore \\ "Department of Neurology, Erasmus University Medical Centre, Rotterdam, The Netherlands
}

Supporting Information

ABSTRACT: Multiple Sclerosis (MScl) is a neurodegenerative disease of the CNS, associated with chronic neuroinflammation. Cerebrospinal fluid (CSF), being in closest interaction with CNS, was used to profile neuroinflammation to discover disease-specific markers. We used the commonly accepted animal model for the neuroinflammatory aspect of MScl: the experimental autoimmune/allergic encephalomyelitis (EAE). A combination of advanced ${ }^{1} \mathrm{H}$ NMR spectroscopy and pattern recognition methods was used to establish the metabolic profile of CSF of EAE-affected rats (representing neuroinflammation) and of two control groups (healthy and peripherally inflamed) to detect specific markers for early neuroinflammation. We found that the CSF metabolic profile for neuroinflammation is distinct from healthy and peripheral inflammation and characterized by changes in concentrations of metabolites such as creatine, arginine, and lysine. Using these disease-specific markers, we were able to detect early stage neuroinflammation,

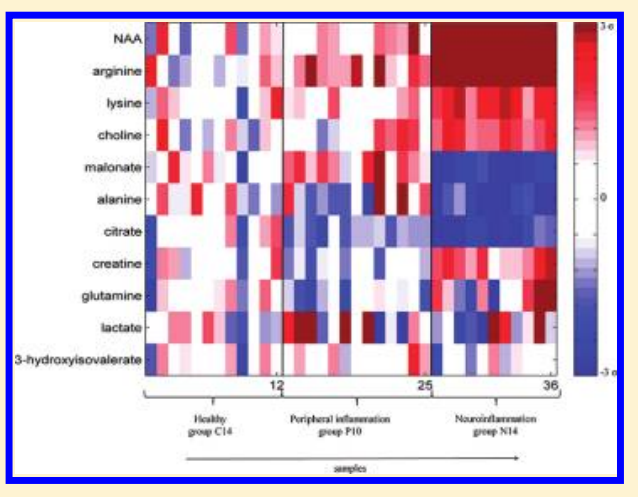
with high accuracy in a second independent set of animals. This confirms the predictive value of these markers. These findings from the EAE model may help to develop a molecular diagnosis for the early stage MScl in humans.

KEYWORDS: ANOVA-PCA, cerebrospinal fluid, EAE model, ${ }^{1} \mathrm{H}$ NMR spectroscopy, pattern recognition, PLS-DA

\section{INTRODUCTION}

Multiple Sclerosis (MScl) is a chronic progressive inflammatory, presumably autoimmune, disease of the human central nervous system (CNS) in which the fatty myelin sheaths that surround the axons of the neurons of the brain and spinal cord are damaged, leading to demyelination. ${ }^{1} \mathrm{MScl}$ is one of the most common neurological diseases and usually starts in early adulthood and progresses to serious neurological disability. It has an enormous impact on the health care system and economy of different countries. Currently, MScl afflicts approximately one million people worldwide, and the total cost of MScl has been estimated at 12.5 billion $€$ per year. ${ }^{2,3}$

The animal model of MScl, the experimental autoimmune/ allergic encephalomyelitis (EAE) model, ${ }^{4-9}$ has become an important tool for understanding the human disease. EAE is a cell-mediated experimental autoimmune disorder of the CNS and shares its clinical expression and pathological picture with that of MScl. For instance, the Lewis rats in which neuroinflammation is induced by means of myelin basic protein (MBP), as in our study here, display a typical disease curve resembling that of the beginning of MScl relapsing-remitting type (RRMScl). RR-MScl is the most common type of the early stage of MScl. EAE is therefore a useful model for the neuroinflammatory aspect of MScl. Note that EAE does not mimic neurodegeneration and widespread demyelination seen in MScl. ${ }^{10}$ In EAE, demyelination might be present in the ventral root exit and dorsal root entry zone of the spinal cord or even absent. Also, EAE is monophasic, whereas MScl has a random relapsing-remitting or chronic progressive pattern. ${ }^{11}$ EAE has led to development of the hypothesized immunological basis of MScl pathology. ${ }^{12}$ Moreover, the EAE has been instrumental in discovering and developing three of the six currently approved therapies for MScl that diminish its symptoms: Copaxone, Mitoxantrone, and Natalizumab. ${ }^{13,14}$ For instance, Mitoxantrone, a known cancer drug, was tested in EAE and found to have positive effects, which led to its use in relapsing-remitting MScl, slowing its progression into secondary progressive MScl. ${ }^{15}$

Unambiguous current clinical diagnosis of MScl remains difficult, particularly in its early stage, due to the complexity of its pathology and the similarities of these pathologies to that of other neurological diseases/inflammations. Diagnosis in an early stage is important as early intervention appears beneficial to slow down the long-term progression of the disease. ${ }^{16} \mathrm{~A}$ molecular

\section{Received: $\quad$ March 3, 2011}

Published: August 02, 2011 


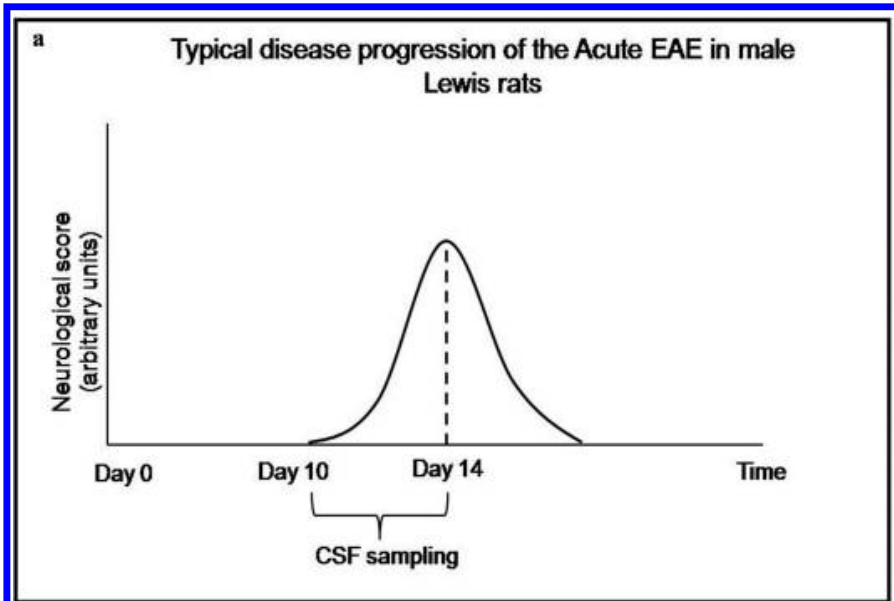

c

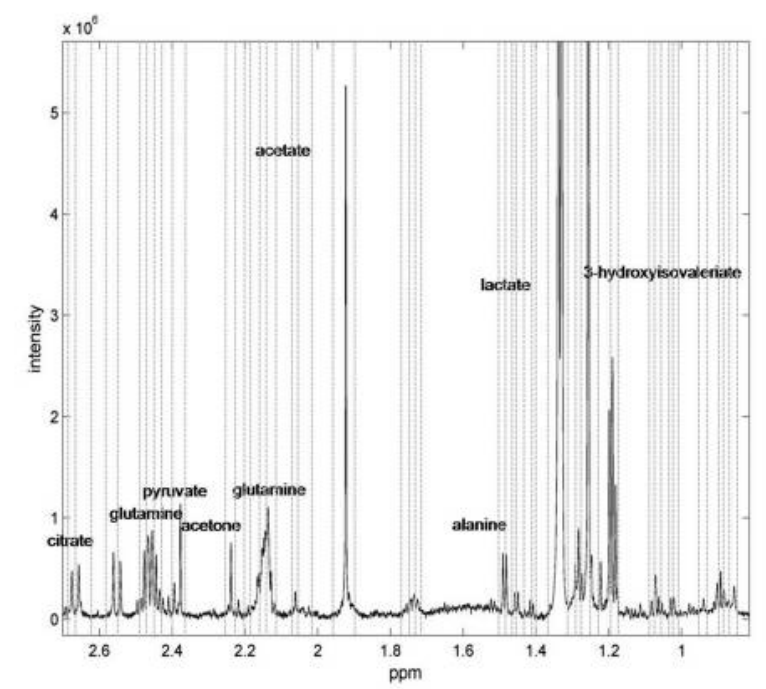

b

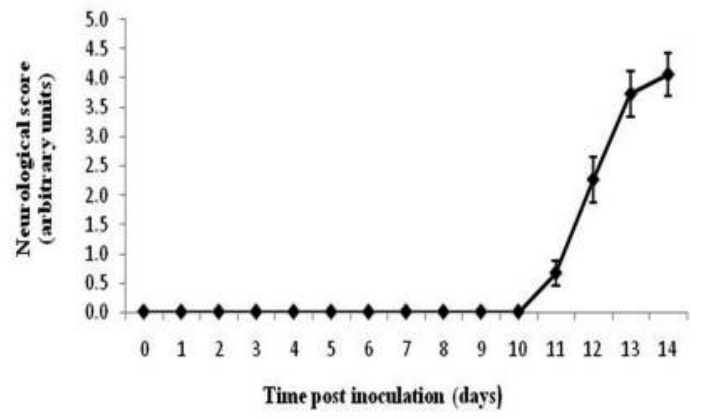

d

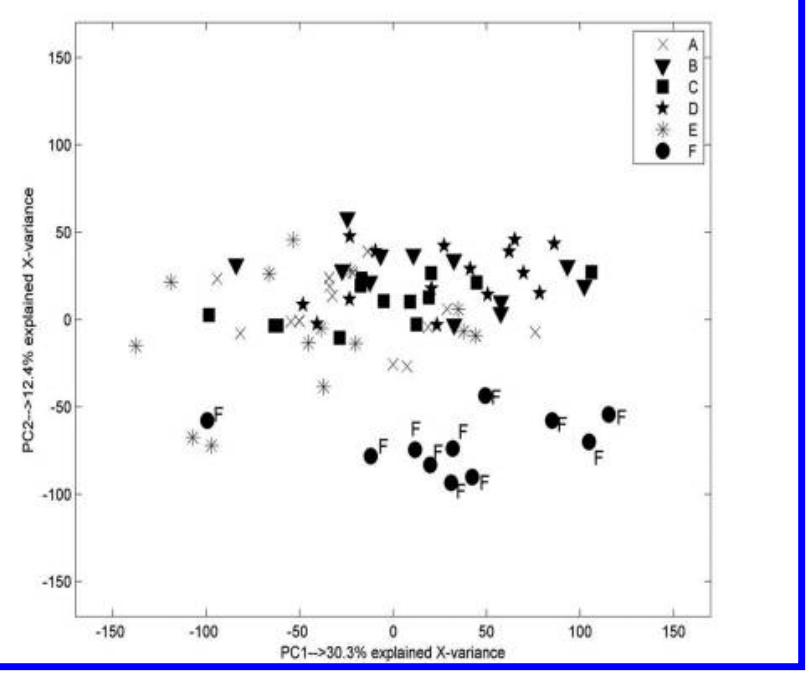

Figure 1. (a) EAE disease progression. (b) Average neurological score screened for group "N14". The vertical bars indicate standard error of the mean. (c) ${ }^{1} \mathrm{H}-800 \mathrm{MHz}$ NMR spectrum of rat CSF. Vertical lines indicate the bins. (d) PCA score plot of the ${ }^{1} \mathrm{H}$ NMR spectra of rat CSF.

biomarker derived for neuroinflammation in the animal model EAE may form a first step toward such an early diagnosis.

The main objective of this study is to find in the cerebrospinal fluid (CSF) of EAE induced Lewis rats metabolic markers of neuroinflammation and to differentiate neuroinflammation from peripheral inflammation. For this a controlled animal study was setup, with a healthy control group, a group injected with inflammatory "booster" (Complete Freund Adjuvant emulsion, CFA) to induce peripheral inflammation and a group injected with CFA and in addition MBP to induce neuroinflammation. Disease progression was monitored, and CSF samples were collected at two different time points. To analyze the metabolic profile of the CSF, we used an untargeted and unbiased biomarker discovery approach in which high-field 1D ${ }^{1} \mathrm{H}$ NMR is combined with pattern recognition methods. To our knowledge, this is the first study applying ${ }^{1} \mathrm{H}$ NMR to analyze rat CSF in the EAE model. A fact that very few rat CSF metabolite studies have been done by NMR is likely due to the limited amount of CSF in rodents. This is not a standard procedure to use such little sample volume in NMR. High quality data could be obtained using only $10 \mu \mathrm{L}$ of CSF, thanks to the use of advanced NMR including high-field and cryoprobe technology.
We found that the CSF metabolic profile for neuroinflammation is distinct from that of healthy and peripheral inflammation. The metabolites, identified as specific for neuroinflammation, were investigated in a second independent set of animals for the prediction of the early stage of neuroinflammation. The validation of the findings with an external experiment is often acclaimed but rarely practiced in a single study. These diseasespecific markers, detected in the animal model, may lead to a better understanding of the metabolism underlying neuroinflammation and help to develop a molecular diagnosis for the early stage of $\mathrm{MScl}$ in humans.

\section{MATERIALS AND METHODS}

\section{Experimental Design of EAE Models}

The EAE is one of the most intensively examined and best characterized animal models of autoimmune disease. ${ }^{9}$ EAE shares similarities with MScl. Although the EAE model does not mimic all aspects of $\mathrm{MScl}$, this rodent disease model is an excellent experimental system for understanding aspects of the MScl disease.

The first set of male Lewis rats (Harlan Laboratories B.V., The Netherlands) was inoculated on Day 0 as previously described. ${ }^{17}$ 
Table 1. Experimental Design of EAE Model ${ }^{a}$

\begin{tabular}{|c|c|c|c|}
\hline treatment day 0 & group description & day 10 & day 14 \\
\hline \multirow[t]{2}{*}{ Anesthesia only } & Healthy & $\mathrm{C} 10$ & $\mathrm{C} 14$ \\
\hline & & $n=15$ & $n=15$ \\
\hline \multirow[t]{2}{*}{ CFA } & Peripheral inflammation & P10 & P14 \\
\hline & & $n=15$ & $n=15$ \\
\hline \multirow[t]{2}{*}{$\mathrm{CFA}+\mathrm{MBP}$} & Neuroinflammation + peripheral & $\mathrm{N} 10^{b}$ & $\mathrm{~N} 14^{c}$ \\
\hline & inflammation & $n=15$ & $n=15$ \\
\hline
\end{tabular}

Briefly, a $100 \mu \mathrm{L}$ saline based emulsion containing $50 \mu \mathrm{L}$ of Complete Freund Adjuvant H37 RA (CFA, Difco Laboratories, Detroit, MI), $500 \mu \mathrm{L}$ of Mycobacterium tuberculosis type H37RA (Difco), and $20 \mu \mathrm{g}$ guinea pig myelin basic protein (MBP) was injected subcutaneously in the pad of the left hind paw of isoflurane anaesthetized animals. CFA was injected for boosting of the immune system, while MBP was injected for induction of neuroinflammation. Next to these MBP challenged rats, referred to as the EAE group (or neuroinflamed group), two control groups were included: a group of animals receiving the same emulsion without MBP (CFA group = peripheral inflammation $)$ and a healthy group undergoing anesthesia only (healthy control). Each group consisted of 30 animals. In each group, half of the animals was sacrificed to collect CSF on Day 10 (Day of onset in EAE group) and the other half on Day 14 (peak of disease in EAE group). The typical progression of disease is shown in Figure 1, while the design of the first EAE experiment is summarized in Table 1.

Animals were kept under normal housing conditions with water and food and libitum, weighing between 175 and $225 \mathrm{~g}$ at the start of the experiment. Animals were group housed per 3 and cages were randomized across treatments and disease duration.

A second set of male Lewis rats was used to perform another EAE experiment, one year after the first one. The animals were inoculated with CFA and/or MBP as described above. The experimental design, the amount of animals (i.e., 15 animals per group) and settings of this model were similar to the first one. The experimental design and the number of animals per group are summarized in Table 1 in the Supporting Information.

Data Acquisition and Analysis

Neurological Scores. Disease symptoms and weights of all animals from both EAE models were recorded daily. The following scores for motor dysfunctions were used: 0 , healthy animal with normal curling reflex at the tail; 1 , paralysis of the tip of the tail; 2, loss of muscle tone at the base of the tail; 3, low posture of hind limbs; 4, instability at hips; 5, partial hind limb paralysis; 6, complete hind limb paralysis; 7 , paralysis include midriff; 8, quadriplegia; 9, moribund; 10, death due to EAE (Supporting Information). All experimental procedures were approved by Abbott's Institutional Animal Care and Use Committee.

CSF Sampling, Sample Preparation and Data Acquisition. On Day 10 and 14, animals were euthanized with $\mathrm{CO}_{2} / \mathrm{O}_{2}$. Terminal CSF samples were obtained by direct insertion of an insulin syringe needle (Myjector, 29G $\times 1 / 2^{\prime \prime}$ ) via the arachnoid membrane into the Cisterna Magna. For this purpose, a skin incision was made followed by a horizontal incision in the musculus trapezius pars descendens to reveal the arachnoid membrane. A maximum volume of $60 \mu \mathrm{L}$ of CSF was collected per animal. Each sample was centrifuged within $20 \mathrm{~min}$ after sampling, for $10 \mathrm{~min}$ at $2000 \times g$ at $4{ }^{\circ} \mathrm{C}$. After centrifugation, the supernatants were stored at $-80^{\circ} \mathrm{C}$ for further analysis. Previous experiments have shown that collecting up to $60 \mu \mathrm{L}$ using this technique under these conditions provides hemoglobin-free CSF samples as measured by ESI-Orbitrap (unpublished data). As an additional check, fresh samples, supernatant, and pellet size were visually scored for hemolysis and samples were discarded if positive.

From the set of 90 samples from the first EAE experiment (Table 1), 4 were contaminated with blood therefore they were excluded from the measurements. A set of 86 CSF samples were prepared and measured as described below.

$10 \mu \mathrm{L}$ of rat CSF were thawed at room temperature and $240 \mu \mathrm{L} \mathrm{D}_{2} \mathrm{O}(99.96$ at.\%D) were added to the biofluid in order to obtain sufficient amount of sample for the NMR measurement. TSP-d4 (Sodium 3-(trimethylsilyl)propionate-2,2,3,3-d4) (99 at.\%D) was used as internal standard for chemical shift reference $(\delta 0.00 \mathrm{ppm}$ ). For the latter, $25 \mu \mathrm{L}$ of $8.8 \mathrm{mM}$ TSP-d4 stock solution in $\mathrm{D} 2 \mathrm{O}$ was added to $250 \mu \mathrm{L}$ of rat CSF to a final concentration of $0.8 \mathrm{mM}$ TSP. The TSP-d4 stock was prepared by weighing in dry TSP-d4. The $\mathrm{pH}$ of the CSF was adjusted to around $7(7.0-7.1)$ by adding phosphate buffer $(9.7 \mu \mathrm{L} 1 \mathrm{M}$, to a final concentration of $35 \mathrm{mM}$ ). The final CSF NMR sample $(284.7 \mu \mathrm{L})$ was then transferred to a SHIGEMI microcell tube for measurements.

The $1 \mathrm{D}{ }^{1} \mathrm{H}$ NMR spectra of rat CSF samples were acquired on an $800 \mathrm{MHz}$ Inova (Varian) system equipped with a $5 \mathrm{~mm}$ tripleresonance, Z-gradient HCN cold-probe. Suppression of water was achieved by using WATERGATE (delay: $85 \mu \mathrm{s}$ ). ${ }^{18}$ For each 1D ${ }^{1} \mathrm{H}$ NMR spectrum, 512 scans were accumulated with a spectral width of $9000 \mathrm{~Hz}$ resulting in a total of $18 \mathrm{~K}$ points. The acquisition time for each scan was $2 \mathrm{~s}$. Between scans, a $8 \mathrm{~s}$ relaxation delay was employed. Prior to spectral analysis, all acquired Free Induction Decays (FIDs) were zero-filled to $32 \mathrm{~K}$ data points, multiplied with a $0.3 \mathrm{~Hz}$ line broadening function, Fourier transformed, manually phased and the TSP internal reference peak was set to $0 \mathrm{ppm}$ by using ACD/SpecManager software version 11.0. ${ }^{19}$ All 86 rat CSF spectra were acquired and preprocessed as described above. However, due to high line broadening of the internal standard (TSP), four spectra were not included in spectral analysis. In total, 82 spectra were subsequently transferred to Matlab, version 7.6 (R2008b) (Mathworks, Natick, MA), for further analysis.

The preparation and data acquisition of CSF samples from the second EAE experiment are described in the Supporting Information.

Preprocessing of NMR Spectra. The NMR spectral data were preprocessed, which typically involves baseline correction, alignment, binning, normalization and scaling. Baseline correction of NMR spectra was performed by applying Asymmetric Least Squares method. ${ }^{20}$ Fluctuation in experimental conditions like sample temperature, $\mathrm{pH}$, ionic strength can lead to chemical shift variations, therefore NMR spectra were aligned by using improved parametric time warping (I-PTW). ${ }^{21} \mathrm{~A}$ further problem is the high dimensionality of the data (ca. 10000 variables). It is common to apply binning to this kind of data, which reduces the number of variables. To perform proper spectral bucketing we used adaptive intelligent binning. ${ }^{22}$ The chemical shift range $\delta 0.75-4.15$ was used for the binning procedure because it contained relevant information. Next, spectral resonances corresponding to one 
metabolite were summed and regrouped in one bin. This procedure was applied to the resonances where no overlapping was present and it led to 153 bins in total. To make spectra comparable between different samples, the final step of preprocessing consisted of integral normalization and supervised vast scaling applied to the binned data. ${ }^{23}$

Metabolites Identification and Quantification. Metabolite identification was carried out by using the $800 \mathrm{MHz}$ library of metabolite NMR spectra from the Chenomx NMR Suite 5.1 ( $\mathrm{pH}$ 6-8). The library of metabolite spectra is obtained based on a database of pure compound spectra acquired using a particular pulse sequence and acquisition parameters, the tn-noesy-presaturation pulse sequence with $4 \mathrm{~s}$ acquisition time and $1 \mathrm{~s}$ of recycle delay. ${ }^{24}$ The Chenomx NMR Suite software fits the spectral signatures (singlets, doublets, triplets etc), that is, the peak shapes, of a compound from an internal database of reference spectra to the experimental NMR spectrum.

For quantification, that is determination of the concentrations of individual metabolites, Chenomx NMR Suite 5.1 uses the concentration of the known reference signal as calibration (in this case TSP-d4). Note that the Chenomx approach is that peak shapes are fitted to the experimental ones. In contrast, peak integration, which is often employed for quantification, is very sensitive to baseline distortions and even slightly overlapping resonances cannot reliably be integrated. Peak-shape fitting, like employed in Chenomx, is instead not much affected by baseline distortions. Moreover, even when some of the resonances and/or part of a resonance signature (triplet etc.) of a compound overlap with that of another compound, the peak shape can still be fitted with reasonable accuracy and the concentration of the compound reliably determined.

Data Analysis Strategy. Explorative analysis, by means of Principal Component Analysis (PCA), ${ }^{25}$ was used first to extract and display the systematic variation in the data.

The strategy for multivariate supervised analysis, using Discriminant Analysis by Projection on Latent Structures (PLS$\mathrm{DA})^{26}$ was designed in accordance with the experimental design summarized in Table 1. The data contain three main groups (healthy control "C10" and "C14", peripheral inflammation "P10" and "P14" and mixture of neuroinflammation and peripheral inflammation "N10" and "N14") in two time points (Day 10 and Day 14). The two time points (Day 10 and Day 14) were analyzed separately, and we always differentiated between two groups. We have used PLS-DA, since it gives more interpretable results and it enabled us to observe the influence of single effects (i.e., peripheral inflammation, neuroinflammation or diseaseprogression) on the CSF metabolic profile.

To corroborate the results we used another supervised method, ANalysis Of VAriance-Principal Component Analysis (ANOVAPCA). The analysis was performed on each time point separately as well as on the two time points combined. Variables (metabolites) differentially profiled across groups of interest were selected based on regression coefficients in PLS-DA and on ANOVA-PCA, Hotelling $\mathrm{T}^{2}$ statistic with $p$-value inferior than $0.05 .^{27}$

Model Construction and Validation. PLS-DA is a variation of PLS. ${ }^{28}$ PLS-DA uses the group information to maximize the separation between groups of observations. It is currently widely used in metabolomics because of its ability to cope with high correlations between variables. In PLS-DA a linear model is constructed according to eq 1 :

$$
\mathbf{y}=\mathbf{X b}+\mathbf{r}
$$

where, $\mathbf{X}$ is a data set matrix, $\mathbf{y}$ is a vector of group memberships, $\mathbf{b}$ is a vector of regression coefficient, and $\mathbf{r}$ is a vector of model residuals. The regression coefficients reflect the relative importance of the variables in the PLS-DA model. We divided the data into a training and a test set using the Duplex algorithm ${ }^{29}$ in such a way that the number of samples in the training set was equal for every considered group. The amount of samples in the test set was equal to at least $25 \%$ of the total number of samples but not more than $30 \%$. To prevent model overfitting we applied the cross model validation (CMV) procedure, introduced by Anderssen et al and Gidskehaug et al. ${ }^{30,31}$ in which double cross validation procedures are included for model optimization, the variable selection based on jack-knifing and final model performance assessment. All MATLAB routines for performing variable selection can be found in this reference. ${ }^{31}$

Finally, all PLS-DA models were validated with the independent test set. For every considered model the specificity and sensitivity of the test set were calculated. The final PLS-DA model was applied to the whole data set if the accuracy of the independent test set was satisfactory (i.e., above $90 \%$ of correct classification).

In order to predict the class labels $(\hat{\mathbf{Y}})$ of validation set the scaled spectra $\left(\mathbf{X}_{\text {new }}\right)$ have to be multiplied by regression coefficient (b) obtained from PLS-DA model (i.e., $\hat{\mathbf{Y}}=\mathbf{X}_{\text {new }} \mathbf{b}$ ). Please keep in mind that scaling performed on validation set applied all necessary parameters (i.e., mean and standard deviation) from training set.

ANOVA-PCA. Previously we showed that this approach allows identifying the relevant variables to distinguish groups. ${ }^{27}$ In this study the main factors are the metabolites effect $(M)$ and the treatments effect (T) (groups) plus a random dimension linked to the individual variations. The ANOVA model is given in eq 2:

$$
\mathrm{X}_{\mathrm{ijk}}=\mu++\mathrm{M}_{\mathrm{i}}+\mathrm{T}_{\mathrm{j}}+\mathrm{MT}_{\mathrm{ij}}+\mathrm{e}_{\mathrm{ijk}}
$$

where $\mu$ is a general mean, e is the error term and $\mathrm{MT}_{\mathrm{ij}}$ indicates the interactions between main effects. The interaction between metabolites and treatments, that is, groups $\left(\mathrm{MT}_{\mathrm{ij}}\right)$, is of highest interest because it reflects the influence of the different treatments on the intensity of metabolites when all others effects are averaged out. Therefore PCA is performed on the matrix of interaction effects to identify metabolites that contribute to this interaction. If the treatment $(j)$ is relevant for the metabolite (i), the interaction $\mathrm{MT}_{\mathrm{ij}}$ is significantly different from zero.

Heat Map and Correlation Network Map. To represent the relevant metabolites concentrations in groups "C14", "P10" and "N14" we generated a heat map from NMR metabolomics data. The heat map is a graphical representation of NMR data in two-dimensional map, where the metabolites concentrations are illustrated as colors. Every concentration value was standardized according to the reference group (healthy group "C14"), i.e. by subtracting mean and dividing by the standard deviation of healthy controls. In that way the metabolites concentrations are expressed in values of standard deviation from the control group.

The concentration of a certain metabolite does not fluctuate independently, but may be correlated and change with the others metabolites. To examine the level of observed changes among metabolites we calculated the Spearman's correlation between relative metabolites concentrations in groups "C14" and "N14", as well in groups "P10" and "N14". Spearman correlation 
calculates the correlation of the ranks for metabolites concentrations, given in eq 3 .

$$
C_{\mathrm{r}}=1-\frac{6 \sum_{i=1}^{n} d_{i}^{2}}{n\left(n^{2}-1\right)}
$$

where, $C_{\mathrm{r}}$ is a Spearman's correlation, $d_{i}$ is a difference between ranks of each sample on the two metabolites, and $n$ is the number of samples.

Extreme variations in concentration values have less influence on the Spearman correlation than on the Pearson's correlation. The calculated correlations are then transferred to Graphviz 1.01 for network map visualization (http://www.graphviz.org). In the network map two metabolites are connected with a link if their correlation coefficient is relevant, that is, exceeds a given threshold. The correlation between metabolites concentrations was significant if its value was superior than 0.7 and $p$-value was inferior than 0.05 .

\section{RESULTS}

\section{Overview of the Available Data}

Eighty-six NMR spectra were measured, while 82 spectra were included in further analysis. An example of a ${ }^{1} \mathrm{H}$ NMR spectrum of rat CSF is shown in Figure 1c in the chemical shift region between 0.8 and $2.7 \mathrm{ppm}$. This region contains several signals from carboxylic acids and amino acids protons. Some of the metabolites are named in Figure 1c. For the analysis, the NMR spectra were divided into 153 bins, which contain resonances of 33 identified metabolites and some unidentified signals. The identified metabolites are listed in the Supporting Information. The information concerning the second EAE model can be found in the Supporting Information.

The average neurological scores and standard deviations for group "N14" are shown in Figure 1b, the information of each individual animal is listed in the Supporting Information. As can be observed, the animals showed motor dysfunction at day 11 . The average day of onset was $11.56( \pm 0.2)$. This means that on average, animals were disabled for a period of 2.4 days $( \pm 0.2)$ at the sampling day. In group "N14" only one animal showed disease duration shorter than 2 days (onset on day 13). The maximum score reached was on average $4.5( \pm 0.2)$. Six animals showed a maximum score above this average. The average peak day (as defined by the first day of maximum score per individual animal) was $13.3( \pm 0.2)$. An idea would be to use the neurological scores for regression purpose for groups "N10" and "N14". However all animals in group "N10" had neurological scores equal zero, while animals in group "N14" quite spread values from 0 until 6. This would cause an artificial compactness of samples from group "N10", while group "N14" very spread. This would lead to biased PLS results. Therefore, the neurological scores were not used.

\section{Explorative Analysis by PCA}

Before performing pattern recognition, the spectra of 82 rat CSF samples were checked for outliers. In total 3 spectra were detected as outliers and were removed from the final analysis. Figure $1 \mathrm{~d}$ shows the PCA score plot of the total data set (79 vast scaled spectra). This plot shows that the samples belonging to group "N14" are clearly separated from the others samples along PC2. It also reveals that a large source of variance in the data does not correspond to the available groups. No clear grouping is present since most of the groups overlap. With PCA only, we are not able to distinguish all analyzed groups. Therefore, we needed to use more dedicated methods.

\section{Supervised Multivariate Analysis by PLS-DA and ANOVA-PCA}

PLS-DA of ${ }^{1} \mathrm{H}$ NMR CSF Data. PLS-DA models of the ${ }^{1} \mathrm{H}$ NMR spectra were performed to extract information on the metabolic effects of the different group treatments as presented in Table 1. In Table 2 in the Supporting Information, the specificity, sensitivity, the correct classification rate for the test set and the total number of variables in the PLS-DA models are presented. Metabolites that contributed significantly to the group separation are listed in Table 3 in the Supporting Information. The results of these PLS-DA models urged us to revise the assumed effects for some groups of Table 1. The summary of the particular effects investigated by the different PLS-DA models is presented in Table 4 in the Supporting Information. As the first part of supervised analysis the comparison between " $\mathrm{C} 10$ " and "C14" has been performed in order to find the metabolites that represent metabolic evolution over days. Later, this information was used to check if any of the discriminating metabolites are related to metabolic variation over time. Following, a detailed analysis of the results of the different models is given.

The score plots of final PLS-DA models are presented in Figures $2 \mathrm{a}-\mathrm{e}$. Note that score plots are used only for illustration purpose and do not determine the classification of PLS-DA model.

Effect of Neuroinflammation and Peripheral Inflammation. The PLS-DA model for groups "C10" versus "N10" allows one to study the combined effect of neuroinflammation and peripheral inflammation. This model has a high prediction ability of $100 \%$ for the independent test set. The area under the curve (93.4\%) indicates that group "C10" and group "N10" are well separated and the PLS-DA has a high model performance. The CSF metabolic profile of the EAE-affected group can thus without a doubt be differentiated from the metabolic profile of the healthy group.

Effect of Neuroinflammation. The PLS-DA model for groups "P10" versus "N10" allows one to discriminate the effects of peripheral inflammation and neuroinflammation on the CSF metabolic profile. This model shows poor prediction (accuracy for the independent test set is $62.5 \%$ ), and the sensitivity for group "N10" is very low (predicted as belonging to group "P10"). The score plot (Figure 2b) illustrates two subgroups in group "N10", one overlapping with group "P10" and a second cluster further away. This result suggests that animals in group "N10" are heterogeneous regarding to the disease response. Probably, within group "N10" some animals did not show any neuroinflammation yet; indeed at Day 10, no neurological deficits have been observed in this particular experiment. Therefore, for some animals the peripheral inflammation is still the dominant effect since the neuroinflammation has not developed yet. A hierarchical clustering was performed on group "N10" to confirm the heterogeneity of the group (data not shown). Three samples were identified as outliers for group "N10". This suggests that the response of the animals to the disease in the EAE model was not uniform and some animals' response is shifted along time. The neurological scores recorded for group "N10" on average were equal to 0 . This means that by examination of the rat's motor system no external neurological or pathological physical signs were observed. The heterogeneity of group "N14" was further investigated with ROC curve analysis. The area under curve (60\%) shows that group "P10" and group "N10" are not well separated. 


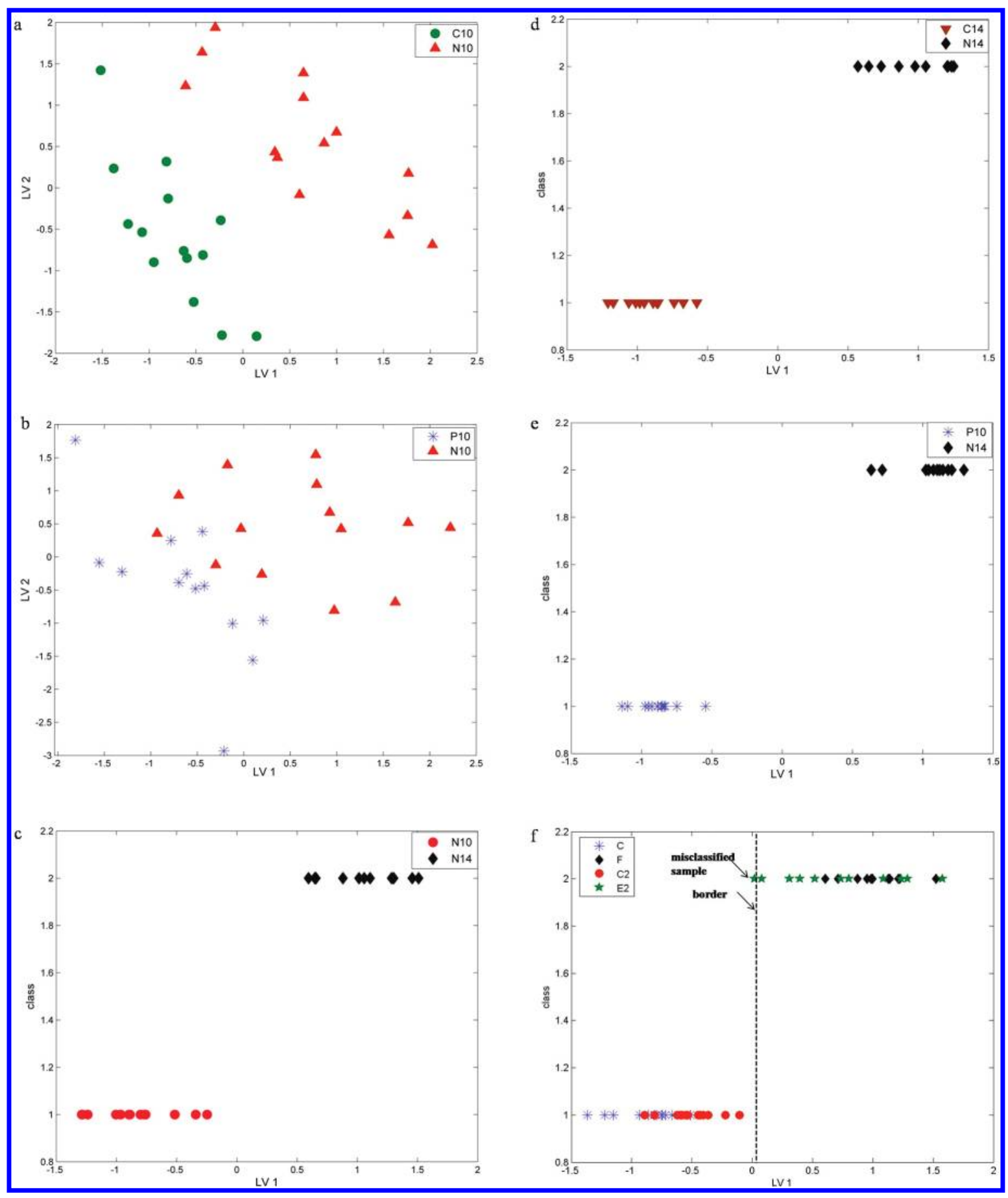

Figure 2. PLS-DA score plots derived from ${ }^{1} \mathrm{H}$ NMR spectra of rat CSF belonging to: (a) groups "C10" and "N10". The amount of Y explained variance for two latent variables was equal to 87.9\%; (b) groups "P10" and "N10". The amount of explained variance in Y for two latent variables was equal 58.3\%; (c) groups "N10" and "N14". The amount of explained variance in Y for one latent variable was equal to 89.6\%; (d) groups "C14" and "N14". The amount of explained variance in $\mathrm{Y}$ for one latent variable was equal to $95.2 \%$; (e) groups "P10" and "N14". The amount of explained variance in $\mathrm{Y}$ for one latent variable was equal to $97.1 \%$; (f) The projection of the independent samples from the second EAE model (group "P10-2" and group "N10-2") on the PLS-DA score plot derived from group "P10" versus "N14" of the first EAE model.

The PLS-DA model for groups “N10” versus "N14” allows one to study the progression of disease. The classification model has perfect accuracy (overall classification of $100 \%$ for independent test set).

Investigation of the second time point (Day 14) by PLS-DA allows one to identify the metabolites differently profiled during the peak of disease. The PLS-DA model of "C14" versus "N14" has perfect prediction ability (overall classification is $100 \%$ for independent test set).

Effect of Peripheral Inflammation during the Peak of the Disease. The overall correct classification rate for independent test set of this model is only $65 \%$. The area under the ROC curve 
Table 2. Average Absolute Concentration $\left(C_{\mu}\right)$ and Standard Deviation $(\sigma)$ of Metabolites Important for Peripheral Inflammation or Neuroinflammation Selected based on PLS-DA and ANOVA-PCA

\begin{tabular}{|c|c|c|c|c|c|c|c|c|}
\hline \multirow[b]{3}{*}{ metabolites } & \multicolumn{8}{|c|}{ groups } \\
\hline & \multicolumn{2}{|c|}{ healthy } & \multicolumn{2}{|c|}{ P10 } & \multicolumn{2}{|c|}{ N10 } & \multicolumn{2}{|c|}{ N14 } \\
\hline & $C_{\mu}[\mu \mathrm{M}]$ & $\sigma[\mu \mathrm{M}]$ & $C_{\mu}[\mu \mathrm{M}]$ & $\sigma[\mu \mathrm{M}]$ & $C_{\mu}[\mu \mathrm{M}]$ & $\sigma[\mu \mathrm{M}]$ & $C_{\mu}[\mu \mathrm{M}]$ & $\sigma[\mu \mathrm{M}]$ \\
\hline NAA $^{a}$ & 13.5 & 2.5 & 14.8 & 1.7 & 16.5 & 2.3 & 21.0 & 3.6 \\
\hline arginine & 39.0 & 10.5 & 37.5 & 8.4 & 46.2 & 11.8 & 62.3 & 17.8 \\
\hline lysine & 72.5 & 9.6 & 68.5 & 11.6 & 89.0 & 15.0 & 135.6 & 29.5 \\
\hline choline & 5.6 & 1.4 & 5.9 & 1.2 & 8.0 & 1.1 & 10.6 & 1.8 \\
\hline malonate & 120.4 & 17.2 & 126.6 & 17.2 & 122.2 & 33.2 & 40.5 & 10.9 \\
\hline alanine & 51.9 & 5.1 & 55.5 & 7.3 & 51.8 & 8.8 & 34.3 & 4.6 \\
\hline citrate & 200.2 & 21.8 & 143.0 & 21.2 & 145.9 & 37.5 & 105.4 & 23.5 \\
\hline creatine & 70.6 & 9.3 & 69.1 & 11.2 & 80.1 & 9.1 & 91.3 & 8.7 \\
\hline glutamine & 606.4 & 93.4 & 531.9 & 96.4 & 544.7 & 71.6 & 654.3 & 127.2 \\
\hline lactate & 2660 & 551.9 & 2757.3 & 387.2 & 3723.0 & 671.8 & 2869.3 & 282.0 \\
\hline 3 -hydroxy ${ }^{a}$ & 494.4 & 17.2 & 430.7 & 25.8 & 439.9 & 33.5 & 510.8 & 15.4 \\
\hline
\end{tabular}

is $59 \%$. These results suggest that the metabolic profiles of group "C14" and group "P14" are quite similar. On the basis of these data, we can make the statement that at Day 14 the effect of an immunopotentiator, that is, CFA, was not measurable based on the metabolite profile. Our interpretation of these results is that peripheral inflammation has vanished by Day 14 and group "P14" is actually healthy. This interpretation is coherent with the absence of external symptoms (i.e., fever and swelling pad). Since group "P14" is not inflamed anymore, the PLS-DA models of group "P14" vs "N14" and "N14" vs ("C14\&P14") give similar results as the PLS-DA model of the healthy group "C14" vs group "N14". In both situations, the overall correct classification for independent test set is $100 \%$. The PLS-DA model of "P10" vs "P14" is described in the Supporting Information.

P10 vs N14: Peripheral Inflammation Effect versus Neuroinflammation Effect. On the basis of the experimental design, the effect in group "N14" is a combination of peripheral inflammation (CFA effect) and neuroinflammation (MBP effect). However, considering the conclusion that at Day 14 the peripheral inflammation has vanished, group "N14" should show only neuroinflammation. Because group "N10" was heterogeneous in disease onset, we used groups "P10" and "N14" to distinguish peripheral inflammation from neuroinflammation. We performed this comparison, knowing that groups "P10" and "N14" represent different conditions, that is, Day 10 for group "P10" and Day 14 for group "N14". This comparison should yield the metabolites influenced by peripheral inflammation or neuroinflammation.

The "P10" vs"N14" PLS-DA model should carry the information about the metabolites differentially profiled in peripherally inflamed and neuroinflamed subjects. The PLS-DA model shows perfect prediction ability with an overall correct classification of $100 \%$ for independent test set. On the basis of our results, these two groups indeed have different metabolic profiles.

ANOVA-PCA of ${ }^{1} \mathrm{H}$ NMR CSF Data. To confirm the results from the PLS-DA models, we used ANOVA-PCA as a corroborative technique to identify metabolites that vary significantly between groups. The factors modeled in this study are the factor treatment (CFA, CFA+MBP or no treatment) and the different metabolites (the Metabolite factor). The resulting biplots of the ANOVA-PCA are displayed in Figure $2 \mathrm{a}-\mathrm{d}$, while the group specific metabolites are summarized in Table 5 in the Supporting Information. In the next section, an overview of the results derived from ANOVA-PCA is discussed.

Except for a few metabolites, the same set of metabolites for peripheral as well as neuroinflammation was selected by ANOVA-PCA. ANOVA-PCA showed that the metabolic profiles of healthy group, peripheral inflamed and neuroinflamed animals can be distinguished. Moreover it showed that groups " $\mathrm{C} 14$ " and "P14" have similar metabolic profiles.

The most discriminating metabolites and their absolute concentrations are presented in Table 2; their short biological interpretation is further discussed in the section Discussion and Conclusion.

As a final step, to make the PLS-DA model more robust and generic (i.e., machine independent), PLS-DA model was constructed for the absolute concentration of the metabolites. This is, however, a time-consuming step, and therefore, we performed it only for the "P10" and "N14" groups. The PLS-DA model for group "P10" versus group "N14" shows perfect prediction ability with an accuracy of $100 \%$ on the independent test set. The score plot of this model is shown in the Supporting Information (Figure 3). This model is further validated with an independent set of animals, derived from a second EAE experiment.

\section{Heat Map and Correlation Network Map}

To represent the individual differences in metabolites concentrations of healthy controls ("C14"), peripherally inflamed ("P10") and neuroinflamed ("N14") animals, a heat map of the metabolomics data was constructed (Figure 3a). The metabolite concentrations were standardized with respect to the healthy group "C14". The metabolites included in the heat map correspond to the significant ones shown in Table 2. The heat map shows that the metabolite concentrations, characteristic for neuroinflammation, change significantly in group "N14". For instance, arginine concentration is elevated in group "N14" in comparison to healthy and peripherally inflamed controls. On the contrary, alanine is reduced. The intraindividual variations are still observable. 


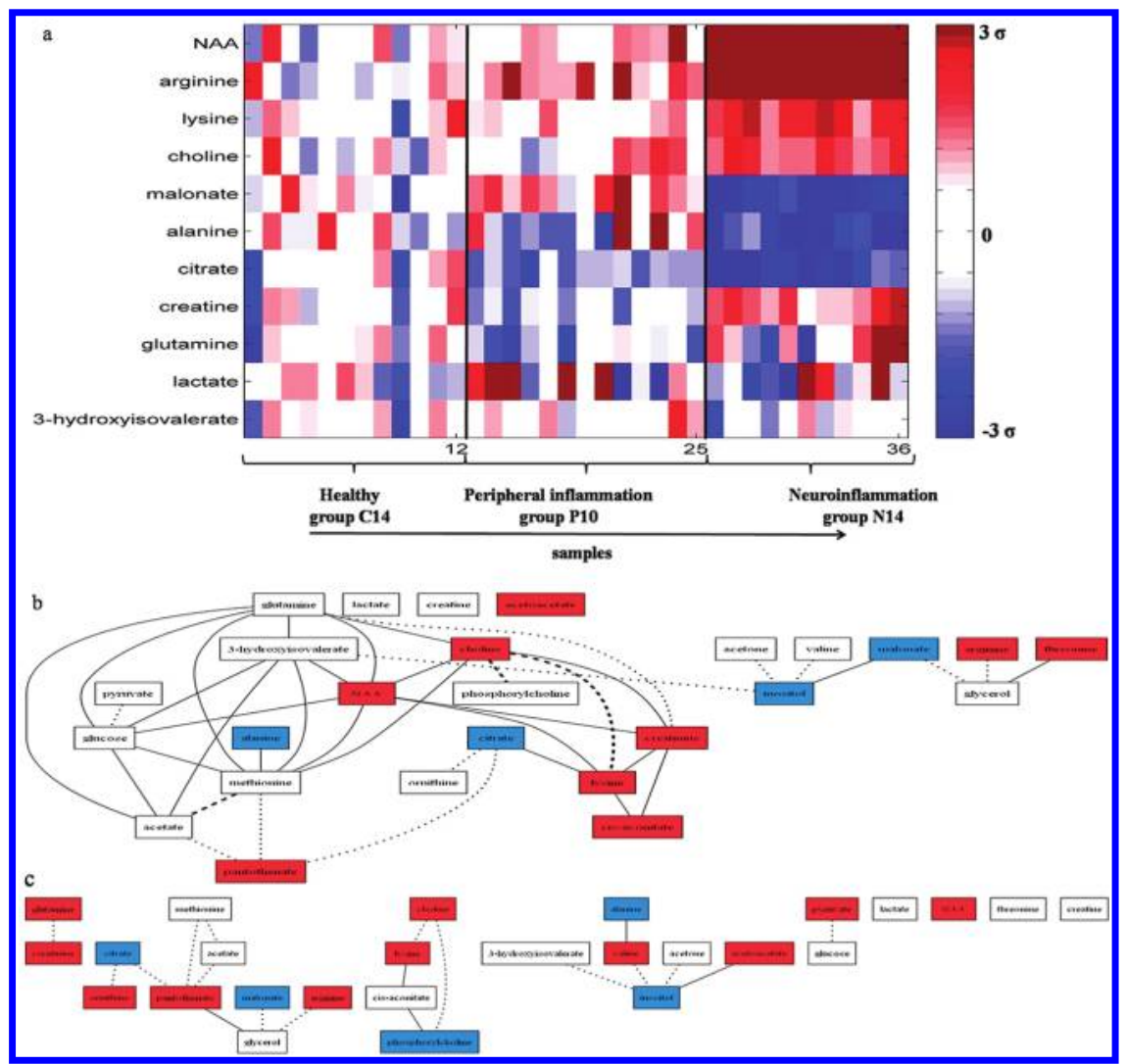

Figure 3. (a) Heat map of selected metabolites for groups "C14", "P10" and "N14". Each row represents a metabolite, and each column corresponds to an animal from healthy group " $\mathrm{C} 14$ " or peripherally inflamed group " $\mathrm{P} 10^{\prime \prime}$, or neuroinflamed group "N14". The relative metabolite concentrations were standardized with respect to healthy group. Red and blue colors represent elevation or reduction of a given metabolite concentration, respectively. NAA stands for $\mathrm{N}$-acetylaspartate, while $\sigma$ indicates the standard deviation of the healthy control. Correlation network map between metabolites identified with high certainty (b) in healthy group " $\mathrm{C} 14$ " and neuroinflamed group "N14" and (c) in peripherally inflamed group "P10" and neuroinflamed group "N14". A solid black line indicates a significant correlation (i.e., correlation $>0.7$ and $p<0.05$ ) occurring in the first group (healthy group "C14" or peripherally inflamed group "P10"). A dotted line signifies the significant correlation unique for neuroinflamed group "N14". A dashed line represents a significant correlation seen in each group. The red and blue boxes represent elevated or reduced metabolite concentrations in neuroinflamed group "N14", respectively. NAA stands for $\mathrm{N}$-acetyaspartate.

To assess the correlation between metabolites, we calculated Spearman's correlation between relative metabolites concentrations in groups "C14" and "N14", as well as in groups "P10" and "N14". Those correlations are visualized as a network map $($ Figure $3 b-c)$ to provide an overview of similarities between different metabolites in the considered groups. In Figure $3 b$, $a$ correlation network map between metabolites in healthy group "C14" and neuroinflamed group "N14" is presented. There are just a few shared correlations (indicated with a dashed line), that is, significant correlations seen in both groups. These correlations are thus not influenced by neuroinflammation. Figure $3 \mathrm{c}$ shows the correlation network map between metabolites in peripherally inflamed group "P10" and neuroinflamed group "N14". There are only a few correlations present in group "P10" (solid, black line), and shared correlations between groups "P10" and "N14" are absent. What is particularly noteworthy is the fact that many correlations, present in the healthy control, vanished due to neuroinflammation, but also some new correlations appeared.

\section{Prediction of Neuroinflammation in an Independent Set of Animals}

To confirm the validity of the discovered disease specific markers for neuroinflammation, we used an independent set of samples coming from another EAE experiment, in which we want to predict neuroinflammation in an early stage. The absolute concentration of our markers was determined for the group with peripheral inflammation at Day 10 (here called group "P10-2") and the group with peripheral and early neuroinflammation at Day 10 (here called group "N10-2"). All peripherally inflamed animals (group "P10-2") are correctly classified. Animals with peripheral inflammation and early neuroinflammation (group "N10-2") are also correctly classified, except for one animal. The overall correct classification for the independent set of samples is $95.8 \%$, with specificity for group "P10-2" and group "N10-2" equal to $100 \%$ and $91 \%$, respectively. The projection of these independent samples on the PLS-DA score plot derived from group "P10" versus "N10" is shown in Figure $2 \mathrm{f}$. It is important to note that the misclassified sample is located close to the borderline between the two groups. This result suggests that proposed markers have a similar behavior in the second study.

\section{DISCUSSION AND CONCLUSION}

Supervised analysis of CSF ${ }^{1} \mathrm{H}$ NMR data revealed significant changes in biochemical composition of the CSF metabolic profile among the analyzed groups. PLS-DA and ANOVA-PCA have been used to model the metabolic profiles from rat CSF. The small differences between PLS-DA and ANOVA-PCA results 
may arise from the principles of the two methods. PLS-DA aims to select a subset of metabolites that gives the highest separations between groups. This may imply that not all discriminatory metabolites are selected. In the case of ANOVA-PCA, the amount of selected variables mostly depend on $\alpha$ level Hotelling $\mathrm{T}^{2}$. In addition, the two different scaling approaches applied before PLS-DA and ANOVA-PCA may have an influence on the selection of metabolites.

Interpretation of PLS-DA models and ANOVA-PCA yielded a set of relevant metabolites, which are shown in Table 2 . In this section we further discuss their biological interpretation

Metabolites Related to Neuroinflammation: Choline, N-Acetylaspartate, Creatine, Lysine, Arginine, Alanine and Malonate

The concentration of choline was elevated in groups "N10" and "N14". However, the choline concentration stayed invariable between two control groups "C10" and "P10". In a previous study, where MScl patients and EAE model in marmoset were studied by ${ }^{1} \mathrm{H}$ NMR spectroscopy, higher choline concentrations have been reported as a marker for demyelination in urine. ${ }^{32}$ This metabolite is required for synthesis of neurotransmitter acetylcholine, and phosphatidylcholine. The increase in choline concentration could be due to demyelination and/or cell membrane breakdown.

Another metabolite found as being highly correlated with EAE-affected groups "N10" and "N14" and that enabled us to differentiate these groups from the others was $N$-acetylaspartate (NAA). This compound is known as a marker of neuronal damage. Its concentration was elevated in the "N14" group. In addition, this metabolite distinguished neuroinflammation from peripheral inflammation in the PLS-DA model. NAA is a free amino acid present in neuronal cell, and it has been culpably involved in many processes of the nervous system, for instance, it may be involved in the myelin production, regulation of neuronal protein synthesis, or the metabolism of several neurotransmitters such as aspartate or $\mathrm{N}$-acetyl-aspartyl-glutamate.

Two other metabolites, creatine and malonate, were found to vary between the two EAE-effected groups. Creatine level was found to be up-regulated in group "N14". Creatine is considered as one of the principal brain metabolite. Its changes in concentration are seen in many other neuro-degenerative disorders and are caused by gliosis ${ }^{33}$ or scarring of neuron (demyelination). An elevated level of creatine in patients with MScl was found in a previous study and has been associated as marker of gliosis. ${ }^{34-36}$ Up-regulated creatine level could be due to a change in the cellular composition, either increased inflammatory cells or glial cells. The malonate level was reduced in neuroinflamed group "N14". Another metabolite found to be reduced in neuroinflamed group "N14" is alanine. Reduced level of alanine in patients with $\mathrm{MScl}$ in comparison to patients with cerebrovascular disease was found in a previous study, where Sinclair et al. used NMR spectroscopy to evaluate the ability of metabolomics analysis to differentiate neurological disease. ${ }^{37}$ Alanine is used as a source for pyruvate for energy metabolism or to synthesis of macromolecules within neural and immune cells. ${ }^{38}$ Reduction of alanine concentration may be connected to energy metabolism, since it might be used by invading cells.

Lysine and arginine were found to have a high correlation with EAE-affected groups "N10" and "N14". Lysine and arginine levels were up-regulated in group "N14" compared to "N10" and in "N10" compared to "P10". This indicates their relation to neuroinflammation. Lysine and arginine are metabolites that may differentiate peripheral inflammation from neuroinflammation. Arginine is used to synthesize nitric oxide (NO). Elevated levels of NO oxidation products in the CNS have been shown in bacterial meningitis in cerebral lupus erythematosus. Recently it was demonstrated that increased levels of $\mathrm{NO}$ oxidation plays a part in the generation of MScl symptoms. ${ }^{39}$ Inhibition of NO synthesis may suppress ${ }^{40}$ or emphasis EAE. ${ }^{41}$ However, the precise role of $\mathrm{NO}$ in $\mathrm{EAE}$ and $\mathrm{MScl}$ still remains elusive and unclear. Qureshi and cowworkers have studied a role of neurotransmitters amino asids in CSF of MScl patients. ${ }^{42}$ They have reported increased level of lysine in CSF in MScl patiens.

Metabolites Related to Peripheral Inflammation: Citrate, Glutamine, Lactate and 3-Hydroxyisovalerate

Citrate is a key metabolite to differentiate the healthy group "C10" from the peripherally inflamed group "P10" but also the peripherally inflamed group from the EAE affected group "N14". Reduced citrate is in line with a previous study of Sinclair and coworkers. ${ }^{37}$ The difference in citrate level is larger between the peripherally inflamed and neuroinflamed group than between the healthy control and the peripherally inflamed group. The citrate level may indicate the degree of inflammation. Citrate is released to a larger extent from astrocytes than from neurons. This metabolite is an intermediate in tricarboxylic acid cycle (TCA). In the study of Smith and co-workers, ${ }^{43}$ the metabolic activity of proteins from myelin and nonmyelin fractions of spinal cords of Lewis rats with EAE was investigated using $\left[1-{ }^{14} \mathrm{C}\right]$ leucine as a protein precursor. In this study, they showed that the decreased activity of the TCA cycle exists. However, the implication of the citrate alternation is unclear although it was already noted in Alzheimer's and in MScl disease. ${ }^{37,44}$

Glutamine is an amino acid, which plays an important role in brain metabolism. This metabolite is involved in energy metabolism. It was shown that glutamine is a necessary nutrient for cell proliferation, serving as a specific fuel for inflammatory cells and enterocytes and, when present in appropriate concentrations, enhancing cell function. During inflammatory states, glutamine consumption may outstrip endogenous production and a relative glutamine deficiency state may exist. ${ }^{45}$

A higher level of lactate was found in group "N10" in comparison to group "N14". Predominant lactate peaks have already been reported in inflammatory CNS diseases. The amount of CSF lactate depends largely on production from CNS glycolysis. Increased lactate production by immune cells is observable in the presence of inflammation. Although elevations in CSF lactate may occur because of many different processes, for instance, hypoxia of inflamed tissues, reduced blood flow from cerebral edema, and granulocyte and bacterial metabolism. ${ }^{46}$ In addition, elevated lactate levels have been identified in vitreous.

The reduced level of 3-hydroxyisovalerate was established for group "N10" and group "P10" when compared with the healthy group. This suggests that this metabolite is involved in peripheral inflammation. However, this result deserves more attention since a previous publication demonstrated an increase in 3-hydroxyisovalerate level in some MScl patients. ${ }^{47}$

\section{Conclusion}

We investigated the effect of neuroinflammation and peripheral inflammation on the metabolic state of CSF in the rat EAE model, a mimic of the neuroinflammatory aspect of the early stage of MScl. In the animal study untargeted and unbiased biomarker discovery approach consisting of high-field $1 \mathrm{D}{ }^{1} \mathrm{H}$ NMR combined with multivariate data analysis was employed. 
CSF is demonstrated as a valuable biofluid for the investigation of neurological disorders in the CNS. We found that ${ }^{1} \mathrm{H}$ NMR is a powerful technique capable of providing information for the identification and quantification of a large number of metabolites in CSF.

The use of two statistical techniques (PLS-DA and ANOVAPCA) contributes significantly to the reliability of the results. The two methods are corroborative, because the overall results obtained by PLS-DA and ANOVA-PCA were found to be coherent.

The CSF metabolic profile for neuroinflammation is distinct from that of healthy and peripheral inflammation and characterized by changes in concentrations of metabolites such as creatine, arginine, and lysine. Peripheral inflammation was only seen at Day 10 and absent at Day 14. A further interesting observation was that the correlation network map is much more complex for the healthy group than for the groups affected by peripheral inflammation or neuroinflammation. Disappearance of correlation between metabolites in peripherally inflamed and neuroinflamed animals might be related to change of penetrability of the blood-brain barrier (BBB). Under standard physiological circumstances, $\mathrm{BBB}$ controls the homeostasis of the interstitial cerebral fluid. ${ }^{48}$ It is known that during EAE changes in the BBB function occur. It causes disruption in the BBB and effects the saturable transport system of substances involved in disease process. $^{49}$ Injection of CFA can itself lead to increased BBB permeability to small molecules and even certain serum proteins. ${ }^{50}$ These disturbances might then cause changes in metabolites flux across $\mathrm{BBB}$ and relations between them. For instance, the correlation might become more nonlinear or weaker and therefore be absent in the disease stage under a selected threshold. In addition, in EAE there is a strong increase in infiltration of the $\mathrm{BBB}$ by monocytes and activated lymphocytes that is bound to change the metabolite profile of the fluids. Not only a disrupted BBB leads to "leakage" but activated immune cells crossing the $\mathrm{BBB}$ and entering the interstitial fluid and the CSF produce metabolites that change the overall profile of the fluids they are in.

Interestingly, some markers of neuroinflammation have been connected to demyelination and neuronal damage. In EAE model induced with MBP demyelination is missing or limited to the ventral root exit and dorsal root entry zone of the spinal cord. In the case of Lewis rats primary demyelination is restricted to occasional perivenous myelin sheaths. In general, demyelination is more distinct when addition of other CNS antigens to the MBP results in pronounced demyelination. In addition, some demyelination was observed in guinea pig EAE model incorporated with $\mathrm{MBP}$ inoculums utilized for sensitization. ${ }^{51}$ However, the presence of demyelination in the EAE model induced with MBP is a matter of debate. Therefore, we believe that it needs additional investigations. In the current study, the presence of demyelination could be investigated by, for instance, repeating the EAE model and then performing histology studies or electron microscopy of spinal cord. Another possibility would be to use Magnetic magnetic resonance imaging to study the pathology of rats' brain and spinal cord. These steps could demonstrate the presence or absence of demyelination.

By using an independent set of animals, that is, coming from another EAE experiment, we demonstrated that this model and ipso facto the disease specific markers have ability to predict neuroinflammation in its early stage with high accuracy. Thus, these animal-model-based markers may be used to diagnose the early stage of neuroinflammation. Further developments will include the investigation and translation of our results to a clinical context, that is, how these results can be used to predict MScl.

\section{ASSOCIATED CONTENT}

\section{S Supporting Information}

Supplementary tables and figures. This material is available free of charge via the Internet at http://pubs.acs.org.

\section{AUTHOR INFORMATION}

\section{Corresponding Author}

*Lutgarde M.C. Buydens, Institute for Molecules and Materials, Analytical Chemistry, Radboud University Nijmegen, Heyendaalseweg 1356525 AJ, Nijmegen, The Netherlands. E-mail: 1. buydens@science.ru.nl. Phone: +(31) 24 3653192. Fax: +(31) 243652653.

Present Addresses

\#Leiden University Medical Center, Leiden, The Netherlands

\section{ACKNOWLEDGMENT}

This study was performed within the framework of Top Institute Pharma project number D4-102.

\section{REFERENCES}

(1) Pilz, G.; Wipfler, P.; Ladurner, G.; Kraus, J. Modern multiple sclerosis treatment - what is approved, what is on the horizon. Drug Discovery Todav 2008, 13 (23-24), 1013-1025.

(2) Sobocki, P.; Pugliatti, M.; Lauer, K.; Kobelt, G. Estimation of the cost of MS in Europe: extrapolations from a multinational cost study. Mult. Scler. 2007, 13 (8), 1054-1064.

(3) Kantarci, O.; Wingerchuk, D. Epidemiology and natural history of multiple sclerosis: new insights. Curr. Opin. Neurol. 2006, 19 (3), $248-254$.

(4) Rivers, T. M.; Sprunt, D. H.; Berry, G. P. Observations on Attempts to Produce Acute Disseminated Encephalomyelitis in Monkeys. I. Exp. Med. 1933, 58 (1), 39-53.

(5) Schwentker, F. F.; Rivers, T. M. The Antibody Response of Rabbits to Injections of Emulsions and Extracts of Homologous Brain. L. Exp. Med. 1934, 60 (5), 559-574.

(6) Kabat, E. A.; Wolf, A.; Bezer, A. E. Rapid Production of Acute Disseminated Encephalomyelitis in Rhesus Monkeys by Injection of Brain Tissue With Adjuvants. Science 1946, 104 (2703), 362-363.

(7) Morgan, I. M. Allergic Encephalomyelitis in Monkeys in Response to Injection of Normal Monkey Nervous Tissue. J. Exp. Med. 1947, 85 (1), 131-140.

(8) Steinman, L.; Zamvil, S. S. How to successfully apply animal studies in experimental allergic encephalomyelitis to research on multiple sclerosis. Ann. Neurol. 2006, 60 (1), 12-21.

(9) Baxter, A. G. The origin and application of experimental autoimmune encephalomyelitis. Nat. Rev. Immunol. 2007, 7 (11), 904-912.

(10) Bradl, M.; Linington, C. Animal models of demyelination. Brain Pathol. 1996, 6 (3), 303-311.

(11) Lublin, F. D. Relapsing experimental allergic encephalomyelitis. An autoimmune model of multiple sclerosis. Springer Semin. Immunopathol. 1985, 8 (3), 197-208.

(12) Wolf, A.; Kabat, E. A.; Bezer, A. E. The Pathology of Acute Disseminated Encephalomyelitis Produced Experimentally in the Rhesus Monkey and Its Resemblance to Human Demyelinating Disease. J. Neuropathol. Exp. Neurol. 1947, 6 (4), 333-357. 
(13) Teitelbaum, D.; Meshorer, A.; Arnon, R.; Sela, M. Suppresion of experimental allergic encephalomyelitis by a synthetic polypeptide. Eur. I. Immunol. 1971, 1, 242-248.

(14) Yednock, T. A.; Cannon, C.; Fritz, L. C.; Sanchezmadrid, F.; Steinman, L.; Karin, N. Prevention of Experimental Autoimmune Encephalomyelitis by Antibodies against Alpha-4-Beta-1 Integrin. Nature 1992, 356 (6364), 63-66.

(15) Ridge, S. C.; Sloboda, A. E.; Mcreynolds, R. A.; Levine, S.; Oronsky, A. L.; Kerwar, S. S. Suppression of Experimental Allergic Encephalomyelitis by Mitoxantrone. Clin. Immunol. Immunopathol. 1985, 35 (1), 35-42.

(16) Tintore, M. Early MS treatment. Int. MS I. 2007, 14, 5-10.

(17) Hendricks, J. J. A.; Alblas, J.; van der Pol, S. M. A.; van Tol, E. A. F.; Dijkstra, C. D.; de Vries, H. E. Flavonoids influence monocytic GTPase activity and are protective in experimental allergic encephalitis. I. Exp. Med. 2004, 200 (12), 1667-1672.

(18) Piotto, M.; Saudek, V.; Sklena, V. Gradient-tailored excitation for single-quantum NMR spectroscopy of aqueous solutions. J. Biomol. NMR 1992, 2 (6), 661-665.

(19) ACD/1D HNMR Manager; Advanced Chemistry Development, Inc: Toronto Ontario, 2003; www.acdlabs.com .

(20) Eilers, P. H. C. A perfect smoother. Anal. Chem. 2003, 75 (14), 3631-3636.

(21) Bloemberg, T. G.; Gerretzen, J.; Wouters, H. J. P.; Gloerich, J.; van Dael, M.; Wessels, H. J. C. T.; van den Heuvel, L. P.; Eilers, P. H. C.; Buydens, L. M. C.; Wehrens, R. Improved parametric time warping for proteomics. Chemom. Intell. Lab. Syst. 2010, 104, 65-74.

(22) de Meyer, T.; Sinnaeve, D.; Van Gasse, B.; Tsiporkova, E.; Rietzschel, E. R.; De Buyzere, M. L.; Gillebert, T. C.; Bekaert, S.; Martins, J. C.; Van Criekinge, W. NMR-based characterization of metabolic alterations in hypertension using an adaptive, intelligent binning algorithm. Anal. Chem. 2008, 80 (10), 3783-3790.

(23) Keun, H. C.; Ebbels, T. M. D.; Antt, H.; Bollard, M. E.; Beckonert, O.; Holmes, E.; Lindon, J. C.; Nicholson, J. K. Improved analysis of multivariate data by variable stability scaling:aplication to NMR-based metabolic profiling. Anal. Chim. ACTA 2003, 490 (1-2), 265-276.

(24) Weljie, A. M.; Newton, J.; Mercier, P.; Carlson, E.; Slupsky, C. M. Targeted profiling: Quantitative analysis of H-1 NMR metabolomics data. Anal. Chem. 2006, 78 (13), 4430-4442.

(25) Trygg, J.; Holmes, E.; Lundstedt, T. Chemometrics in metabonomics. I. Proteome Res. 2007, 6 (2), 469-479.

(26) Giskeodegard, G. F.; Grinde, M. T.; Sitter, B.; Axelson, D. E.; Lundgren, S.; Fjosne, H. E.; Dahl, S.; Gribbestad, I. S.; Bathen, T. F. Multivariate Modeling and Prediction of Breast Cancer Prognostic Factors Using MR Metabolomics. I. Proteome Res. 2010, 9 (2), 972-979.

(27) de Haan, J. R.; Wehrens, R.; Bauerschmidt, S.; Piek, E.; van Schaik, R. C.; Buydens, L. M. C. Interpretation of ANOVA models for microarray data using PCA. Bioinformatics 2007, 23 (2), 184-190.

(28) Westerhuis, J. A.; Hoefsloot, H. C. J.; Smit, S.; Vis, D. J.; Smilde, A. K.; van Velzen, E. J. J.; van Duijnhoven, J. P. M.; van Dorsten, F. A. Assessment of PLSDA cross validation. Metabolomics 2008, 4 (1), 81-89.

(29) Snee, R. D. Validation of regression models: Methods and examples. Technometrics 1977, 19 (4), 415-428.

(30) Anderssen, E.; Dyrstad, K.; Westad, F.; Martens, H. Reducing over-optimism in variable selection by cross-model validation. Chemom. Intell. Lab. Syst. 2006, 84 (1-2), 69-74.

(31) Gidskehaug, L.; Anderssen, E.; Alsberg, B. K. Cross model validation and optimization of bilinear regression models. Chemom. Intell. Lab. Sust. 2008, 93 (1), 1-10.

(32) t'Hart, B. A.; Vogels, J. T.; Spijksma, G.; Brok, H. P.; Polman, C.; van der Greef, J. 1H-NMR spectroscopy combined with pattern recognition analysis reveals characteristic chemical pattern in urines of MS patients and non-human primates with MS-like disease. J. Neurol. Sci. 2003, 212 (1-2), 21-30.

(33) Hattingen, E.; Magerkurth, J.; Pilatus, U.; Hubers, A.; Wahl, M.; Ziemann, U. Combined (1) $\mathrm{H}$ and (31)P spectroscopy provides new insights into the pathobiochemistry of brain damage in multiple sclerosis. NMR Biomed. 2010, 24, 536-546.
(34) Qian, J.; Herrera, J. J.; Narayana, P. A. Neuronal and axonal degeneration in experimental spinal cord injury: in vivo proton magnetic resonance spectroscopy and histology. L. Neurotrauma 2010, 27 (3), 599-610.

(35) Sajja, B. R.; Wolinsky, J. S.; Narayana, P. A. Proton magnetic resonance spectroscopy in multiple sclerosis. Neuroimaging Clin. N. Am. 2009, 19 (1), 45-58.

(36) Sarchielli, P.; Presciutti, O.; Tarducci, R.; Gobbi, G.; Alberti, A.; Pelliccioli, G. P.; Chiarini, P.; Gallai, V. Localized (1)H magnetic resonance spectroscopy in mainly cortical gray matter of patients with multiple sclerosis. L. Neurol. 2002, 249 (7), 902-910.

(37) Sinclair, A. J.; Viant, M. R.; Ball, A. K.; Burdon, M. A.; Walker, E. A.; Stewart, P. M.; Rauz, S.; Young, S. P. NMR-based metabolomic analysis of cerebrospinal fluid and serum in neurological diseases--a diagnostic tool? NMR Biomed. 2010, 23 (2), 123-132.

(38) Noga, M. J.; Dane, A.; Shi, S.; Attali, A.; van Aken, H.; Suidgeest, E.; Tuinstra, T.; Muilwijk, B.; Coulier, L.; Luider, T. M.; Reijmers, T. H.; Vreeken, R. J.; Hankemeier, T. Metabolomics of cerebrospinal fluid reveals changes in central nervous system metabolism in a rat model of multiple sclerosis. Metabolomics 2011; DOI: 10.1007/s11306-011-0306-3.

(39) Danilov, A. I.; Andersson, M.; Bavand, N.; Wiklund, N. P.; Olsson, T.; Brundin, L. Nitric oxide metabolite determinations reveal continuous inflammation in multiple sclerosis. J. Neuroimmunol. 2003, 136 (1-2), 112-118.

(40) Ding, M. Z.; Zhang, M.; Wong, J. L.; Rogers, N. E.; Ignarro, L. J.; Voskuhl, R. R. Cutting edge: Antisense knockdown of inducible nitric oxide synthase inhibits induction of experimental autoimmune encephalomyelitis in SJL/J mice. I. Immunol. 1998, 160 (6), 2560-2564.

(41) Zielasek, J.; Jung, S.; Gold, R.; Liew, F. Y.; Toyka, K. V.; Hartung, H. P. Administration of Nitric-Oxide Synthase Inhibitors in Experimental Autoimmune Neuritis and Experimental Autoimmune Encephalomyelitis. I. Neuroimmunol. 1995, 58 (1), 81-88.

(42) Qureshi, G. A.; Baig, S. M. Role of Neurotransmitter AminoAcids in Multiple-Sclerosis in Exacerbation, Remission and Chronic Progressive Course. Biog. Amines 1993, 10 (1), 39-48.

(43) Smith, M. E.; Rauch, H. C. Metabolic-Activity of Cns Proteins in Rats and Monkeys with Experimental Allergic Encephalomyelitis (Eae). I. Neurochem. 1974, 23 (4), 775-783.

(44) Ghauri, F. Y. K.; Nicholson, J. K.; Sweatman, B. C.; Wood, J.; Beddell, C. R.; Lindon, J. C.; Cairns, N. J. Nmr-Spectroscopy of Human Postmortem Cerebrospinal-Fluid - Distinction of Alzheimers-Disease from Control Using Pattern-Recognition and Statistics. NMR Biomed. 1993, 6 (2), 163-167.

(45) Wilmore, D. W.; Shabert, J. K. Role of glutamine in immunologic responses. Nutrition 1998, 14 (7-8), 618-626.

(46) Watson, M. A.; Scott, M. G. Clinical utility of biochemical analysis of cerebrospinal fluid. Clin. Chem. 1995, 41 (3), 343-360.

(47) Lutz, N. W.; A., V.; Malikova, I.; Confort-Gouny, S.; Ranjeva, J. P.; Cozzone, P. J. A branched-chain organic acid linked to multiple sclerosis:First identification by NMR spectroscopy of CSF. Biochem. Biophvs. Res. Commun. 2007, 354 (1), 160-164.

(48) Kunz,J.; Krause, D.; Gehrmann, J.; Dermietzel, R. Changes in the Expression Pattern of Blood-Brain Barrier-Associated Pericytic Aminopeptidase-N (Pap-N) in the Course of Acute Experimental Autoimmune Encephalomyelitis. I. Neuroimmunol. 1995, 59 (1-2), 41-55.

(49) Pan, W.; Banks, W. A.; Kennedy, M. K.; Gutierrez, E. G.; Kastin, A. J. Differential permeability of the BBB in acute EAE: enhanced transport of TNT-alpha. Am. I. Physiol. 1996, 271 (4 Pt 1), E636-642.

(50) Pan, W.; Banks, W. A.; Kennedy, M. K.; Gutierrez, E. G.; Kastin, A. J. Peripheral injections of Freund's adjuvant in mice provoke leakage of serum proteins through the blood-brain barrier without inducing reactive gliosis. Brain Res. Bull. 1999, 832 (1-2), 84-96.

(51) Linington, C.; Bradl, M.; Lassmann, H.; Brunner, C.; Vass, K. Augmentation of Demyelination in Rat Acute Allergic Encephalomyelitis by Circulating Mouse Monoclonal-Antibodies Directed against a Myelin Oligodendrocyte Glycoprotein. Am. J. Pathol. 1988, 130 (3), 443-454. 